Mathematical Modelling and Analysis

Volume 18 Number 3, June 2013, 386-394

http://dx.doi.org/10.3846/13926292.2013.804890

(c) Vilnius Gediminas Technical University, 2013
Publisher: Taylor\&Francis and VGTU

http://www.tandfonline.com/TMMA

Print ISSN: 1392-6292

Online ISSN: 1648-3510

\title{
Effective Conductivity of 2D Disk - Ring Composite Material
}

\author{
Serhii Gryshchuk ${ }^{a, b}$ and Sergei Rogosin ${ }^{c}$ \\ ${ }^{a}$ University of Padova \\ Via Trieste 63, 35121 Padova, Italy \\ ${ }^{b}$ National Academy of Sciences of Ukraine \\ 3 Tereshchenkivska st., 01601 Kiev-4, Ukraine \\ ${ }^{c}$ Belarusian State University \\ Nezavisimosti ave 4, BY-220030 Minsk, Belarus \\ E-mail: serhii.gryshchuk@gmail.com \\ E-mail(corresp.): rogosinsv@gmail.com
}

Received August 10, 2012; revised May 3, 2013; published online June 1, 2013

\begin{abstract}
For 2D bounded composite material geometrically composed by a disk of variable radius $r$ and an outer ring it is determined in an analytic form the $x$-component of the effective conductivity tensor. Namely, it is shown that the $x$-component is a sum of geometrical progression with respect to powers of $r^{2}$ for all sufficiently small $r$.
\end{abstract}

Keywords: bounded 2D composites, disk-ring, heat conduction, $\mathbb{R}$-linear problem, effective conductivity.

AMS Subject Classification: 74A40; 74G10; 30E25.

\section{Introduction}

Circular type 2D mechanical structures are subject of long investigations. In the pioneering work [9] it had been found a complete solution of the problem of torsion and bending of elastic cylindric body reinforced by parallel cylindric beams $S_{k}, k=1, \ldots, m$, of different materials. The author showed that this problem is equivalent to the problem, which can be formulated in the following form.

Problem $A$. Let $S_{k}, k=0,1, \ldots, m$, are simply connected non-overlapping domains of the complex plane $\mathbb{C}$ with smooth boundaries $C_{0}, C_{1}, \ldots, C_{m}$, such that the contour $C_{0}$ embraces any contour $C_{k}, k=1, \ldots, m$. Let $S:=$ $S_{0} \backslash \cup_{k=1}^{m} S_{k}$. The problem is to find a function $\varphi$ harmonic in each domain $S_{k}$, $k=0,1, \ldots, m$, continuous in $\mathrm{cl} S_{0}$ and such that its normal derivative on each contour $C_{k}, k=0, \ldots, m$ has a jump satisfying given conditions. 
Here and anywhere $\operatorname{cl}(G)$ denotes a closure of a domain $G \subset \mathbb{C}$.

It is proved in [9] that the given problem is equivalent to a system of $m+1$ Fredholm integral equations of the second kind. He showed that under some solvability conditions this system has a solution and this solution is unique up to an additive constant. The main aim of the above article is to calculate certain elastic characteristics of the considered elastic cylindric body reinforced by parallel cylindric beams. One of these is, so-called, elastic torsional rigidity. Mathematically speaking, it is a real valued functional depending on the solution of the latter problem.

The solution of the Problem A is uniquely determined from a system of Fredholm integral equations of the second kind. Anyway, from the practical point of view it is important to find an explicit expression of the solution. Therefore, any solution' method for Problem A which leads to a closed form solution (even in certain special cases), is interesting for the practice. For example, in case when $m=k=1$ and $S_{0}$ and $S_{1}$ are disks (or what is equivalent $S_{0}^{\prime}$ and $S_{1}^{\prime}$ are disks with a common origin) the Problem A, is solved by the direct method based on expansions in Taylor's and Laurent's series of a function $f(z)$, which is analytic in $S_{1}^{\prime} \cup S^{\prime}, S^{\prime}=S_{0}^{\prime} \backslash \mathrm{cl} S_{1}^{\prime}$. The function $f(z)$ is such that its real part Re $f$ equals to an unknown function $\varphi$ in $S_{1}^{\prime} \cup S^{\prime}$. By using this solution the elastic torsional rigidity is calculated in an explicit form in [10]. The case when $m=k=1$ and $S_{0}$ and $S_{1}$ are confocal ellipses is considered in [11]. The discussion on recent contributions to the explicit solution of Problem $\mathrm{A}$ in the case of non-circular geometry is presented in [7].

Our main aim is to solve a problem similar to Problem A when $m=k=1$ and $S_{0}$ and $S_{1}$ are disks with a common origin, geometrically representing a bounded 2D composite material. Here, the annulus $S=S_{0} \backslash \operatorname{cl} S_{1}$ and the disk $S_{1}$ are domains corresponding to the matrix and the inclusion of the composite, respectively. On the base of this solution we calculate an analog of the elastic torsional rigidity, which is an $x$-component of, so-called, Effective Conductivity Tensor (ECT). In spite of the fact, that usually the term "effective conductivity" is used for composite materials with a rich microstructure, we utilize the same term here, meaning effective conductivity of a bounded composite material, see, e.g., $[2,3,5]$.

More precisely, we consider the problem of determination of the temperature distribution under perfect contact condition in the described inhomogeneous media, which is loaded by a simple heat flow. Note, that this problem is equivalent to the $\mathbb{R}$-linear conjugation problem on the complex plane (for more details on the solution of similar problems we refer to $[4,6],[8$, p. 45]). The formula for the $x$-component of the effective conductivity tensor is represented in an analytic form in terms of radius $r$ of the internal disk and so-called contrast parameter $\rho$ introduced by Bergmann [1].

The obtained exact formula allows us to apply it for the description of certain physical phenomenon, these results will be presented in a separate paper. We can to consider also an asymptotic behavior of the solution and components of the effective conductivity tensor in the case when inclusion is either shrinking to a point or expanding up to the whole domain $S_{0}$. 


\section{Formulation of the Problem}

Let $\mathbb{C}$ be the complex plane, and $\mathbb{R}$ be the field of real numbers. For any $\xi$ from $\mathbb{C}$ or $\mathbb{R}$ let us denote by $|\xi|$ its modulus. For any $r \in(0,1)$ we consider the following sets of the complex plane: $\mathrm{D}_{r}:=\{z \in \mathbb{C}:|z|<r\}, \gamma_{r}:=\{t \in \mathbb{C}:|t|=$ $r\}, \mathrm{A}_{r}:=\{z \in \mathbb{C}: r<|z|<1\}, \Gamma:=\{t \in \mathbb{C}:|t|=1\}$. The boundary $\partial \mathrm{D}_{r}$ of $\mathrm{D}_{r}$ is denoted by $\gamma_{r}$ and $\partial \mathrm{A}_{r}:=\gamma_{r} \cup \Gamma$. For any $z=x+i y \in \mathbb{C}$ we denote by $(\eta, \theta), 0<\eta<\infty, 0 \leq \theta \leq 2 \pi$, its polar coordinates, thus, $z=\eta \exp \{i \theta\} ; \operatorname{Re} z$ is a real part of a complex number $z$.

Let $\Omega$ be an open or closed domain in $\mathbb{C}$ and let $\tau: \Omega \longrightarrow \mathbb{R}$ be a real valued function, then $\mathcal{C}^{k}(\Omega), k \in 1, \ldots$, denotes a class of functions having continuous partial derivatives up to $k$-th order. If $\tau \in \mathcal{C}^{2}(\Omega)$ then

$$
\Delta \tau(x+i y):=\frac{\partial^{2} \tau}{\partial x^{2}}+\frac{\partial^{2} \tau}{\partial y^{2}} .
$$

For any function $\varphi: \mathrm{D}_{r} \cup \mathrm{A}_{r} \longrightarrow \mathbb{R}$, by $\varphi_{1}$ and $\varphi_{2}$ we mean its restrictions to $\mathrm{D}_{r}$ and $\mathrm{A}_{r}$, respectively.

Let us consider 2D circular composite material consisting of two components, represented geometrically by the annulus $\mathrm{A}_{r}$ (a host material or matrix) and by the disk $\mathrm{D}_{r}$ (inclusion). Each component of the composite is filled in by the homogeneous material of constant thermal conductivity $\lambda_{2}$ and $\lambda_{1}$, respectively. We suppose that a normalized external heat flow is put in the direction opposite to $x$-axes. Then the main problem is to determine the potential temperature distribution in such a composite in the stationary case.

Mathematically this problem consists in determination of a pair of realvalued functions $\varphi=\left(\varphi_{1}, \varphi_{2}\right)$, with $\varphi_{1} \in C^{2}\left(\mathrm{D}_{r}\right) \cap C^{1}\left(\operatorname{clD}_{r}\right), \varphi_{2} \in C^{2}\left(\mathrm{~A}_{r}\right) \cap$ $C^{1}\left(\mathrm{cl} \mathrm{A}_{r}\right)$ representing temperature distribution in the corresponding domains, satisfying the following system of relations

$$
\begin{cases}\Delta \varphi(z)=0, & \forall z \in \mathrm{D}_{r} \cup \mathrm{A}_{r}, \\ \varphi_{2}(t)=-\operatorname{Re} t, & \forall t \in \Gamma, \\ \varphi_{1}(t)=\varphi_{2}(t), & \forall t \in \gamma_{r}, \\ \lambda_{1} \frac{\partial \varphi_{1}}{\partial \mathbf{n}}(t)=\lambda_{2} \frac{\partial \varphi_{2}}{\partial \mathbf{n}}(t), & \forall t \in \gamma_{r},\end{cases}
$$

where $\lambda_{1}$ and $\lambda_{2}$ are some positive constants such that $\rho:=\frac{\lambda_{2}-\lambda_{1}}{\lambda_{2}+\lambda_{1}}$ does not equal to zero, $\varphi_{1}(t)$ and $\varphi_{2}(t)$ denote the boundary values of, respectively, $\varphi_{1}(z), z \in \mathrm{D}_{r}$, and $\varphi_{2}(z), z \in \mathrm{A}_{r}, \frac{\partial \varphi_{k}}{\partial \mathbf{n}}, k=1,2$, is respectively an outward and inward normal derivatives with respect to the boundary of the domain of the function $\varphi_{k}, k=1,2$. Since by definition domains of the functions $\varphi_{1}$ and $\varphi_{2}$ are $\mathrm{D}_{r}$ and $\mathrm{A}_{r}$, then for any $t \in \gamma_{r}$ the directions of the normal $\mathbf{n}$ at the left and at the right side of the fourth equality of the system (2.1) coincide (see Fig. 1).

Problem (2.1) (cf., e.g., [8, p. 45] with $\lambda_{2}=1$ ) can be reduced to the following $\mathbb{R}$-linear conjugation problem: find two functions $\phi: \mathrm{D}_{\mathrm{r}} \cup \mathrm{A}_{r} \longrightarrow$ $\mathbb{C}, \phi_{0}: \mathbb{C} \cup\{\infty\} \longrightarrow \mathbb{C}, \phi_{0}(\infty)=0$, such that $\phi=\left(\phi_{1}, \phi_{2}\right)$ is analytic in $\mathrm{D}_{r} \cup \mathrm{A}_{r}$ with $\phi_{1}, \phi_{2}$ being continuous in $\mathrm{cl}_{r}$, cl $\mathrm{A}_{r}$, respectively, $\phi_{0}$ is analytic 


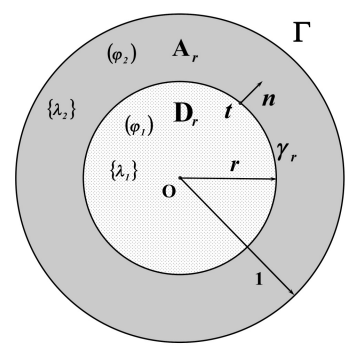

Figure 1. A section of a bounded 2D composite.

in ext $\mathrm{D}_{1}=\{z \in \mathbb{C}:|z|>1\}$ and continuous in $\operatorname{cl}\left\{\operatorname{ext} \mathrm{D}_{1}\right\}$, functions $\phi$ and $\phi_{0}$ satisfy the following conjugation conditions

$$
\phi^{+}(t)=\phi^{-}(t)-\rho \overline{\phi^{-}(t)} \quad \forall t \in \gamma_{r}, \quad \phi^{-}(t)=\phi_{0}(t)-\overline{\phi_{0}(t)}-t \quad \forall t \in \Gamma,
$$

where $\phi^{+}(t)$ and $\phi^{+}(t), t \in \gamma_{r}$, is boundary values of $\phi(z)$, accordingly, from $\mathrm{D}_{r}, \mathrm{~A}_{r}, \overline{u+i v}:=u-i v$ for $u, v \in \mathbb{R}$.

Note, that the problems similar to $(2.2)$ have been considered in $[4,6,8]$. Our aim is to calculate $x$-component of the effective conductivity tensor (see, e.g. [5]), i.e. a quantity $\lambda_{\text {eff }}^{x}$ satisfying the following equality

$$
F^{x} \lambda_{e f f}^{x}(r)=\lambda_{1} \iint_{D_{r}} \frac{\partial \varphi_{1}}{\partial x} d x d y+\lambda_{2} \iint_{\mathrm{A}_{r}} \frac{\partial \varphi_{2}}{\partial x} d x d y
$$

where $\varphi(x, y):=\left(\varphi_{1}(x+i y), \varphi_{2}(x+i y)\right)=\operatorname{Re}\left(\phi_{1}(x+i y), \phi_{2}(x+i y)\right)$ with $\phi_{1}(x+i y), \phi_{2}(x+i y)$ being the solution to $(2.2), F^{x}$ is a complete flux in $x$-direction.

The first step is to find a solution of (2.1) in an explicit form.

\section{Solution of Problem 2.1}

In order to solve boundary value problem (2.1) we propose a method based on expansions in the Taylor's and the Laurent's power series of $f_{1}$ and $f_{2}$. Here $f_{1}$ and $f_{2}$ are restrictions of a function $f(z): \mathrm{D}_{r} \cup \mathrm{A}_{r} \longrightarrow \mathbb{C}$, such that $f \equiv f_{1}$ and $f_{1}$ is analytic in $\mathrm{D}_{r}, \operatorname{Re} f_{1}(z)=\varphi_{1}(x, y)$ for all $z=x+i y \in \mathrm{D}_{r} ; f \equiv f_{2}$ and $f_{2}$ is analytic in $\mathrm{A}_{r}, \operatorname{Re} f_{2}(z)=\varphi_{2}(x, y)$ for all $z=x+i y \in \mathrm{A}_{r}$.

Note, that a similar idea was realized in the paper [10].

Theorem 1. For any $0<r<1$ the solution of the boundary value problem (2.1) is given by the following formulas

$$
\begin{aligned}
& \varphi_{1}(z)=-\eta \frac{1-\rho^{-1}}{r^{2}-\rho^{-1}} \cos \theta \quad \forall z=\eta \exp \{i \theta\} \in \mathrm{D}_{r} \\
& \varphi_{2}(z)=\left(\eta \frac{\rho^{-1}}{r^{2}-\rho^{-1}}-\eta^{-1} \frac{r^{2}}{r^{2}-\rho^{-1}}\right) \cos \theta \quad \forall z=\eta \exp \{i \theta\} \in \mathrm{A}_{r} .
\end{aligned}
$$


Furthermore,

$$
\begin{aligned}
& \varphi_{1}(t)=\varphi_{2}(t)=-\left(1-\rho^{-1}\right) \frac{r}{r^{2}-\rho^{-1}} \cos \theta \quad \forall t=r \exp \{i \theta\} \in \gamma_{r} \\
& \varphi_{2}(t)=-\cos \theta \quad \forall t=\exp \{i \theta\} \in \Gamma
\end{aligned}
$$

Proof. Let a function $f_{1}: \mathrm{D}_{r} \longrightarrow \mathbb{C}$ be an analytic in $\mathrm{D}_{r}$ and such that $\operatorname{Re} f_{1}=\varphi_{1}$ in $\mathrm{D}_{r} \cup \gamma_{r}$. Then the function $f_{1}$ has an expansion in the Taylor's power series in $\mathrm{D}_{r}$ and we have

$$
f_{1}(z)=a_{0}^{\prime}+i b_{0}^{\prime}+\sum_{k=1}^{\infty}\left(a_{k}^{\prime}+i b_{k}^{\prime}\right) z^{k} \quad \forall z \in \mathrm{D}_{r}
$$

Let a function $f_{2}: \mathrm{A}_{r} \longrightarrow \mathbb{C}$ be a single-valued analytic function in $\mathrm{A}_{r}$, such that $\operatorname{Re} f_{2}=\varphi_{2}$ in $\mathrm{A}_{r} \cup \gamma_{r} \cup \Gamma$. Then it can be expanded in the Laurent's series in $\mathrm{A}_{r}$ and the following equality holds

$$
f_{2}(z)=a_{0}^{\prime \prime}+i b_{0}^{\prime \prime}+\sum_{k=-\infty, k \neq 0}^{\infty}\left(a_{k}^{\prime \prime}+i b_{k}^{\prime \prime}\right) z^{k} \quad \forall z \in \mathrm{A}_{r}
$$

It follows from (3.5), (3.6) and the choice of functions $f_{1}$ and $f_{2}$ that the functions in the right-hand side of (3.1) and (3.2) are proper candidates to be solutions of the problem (2.1). We have to check later whether the functions $\varphi_{1}$, $\varphi_{2}$, satisfying conditions (2.1), belongs to considered functional spaces. Taking real part of (3.5), (3.6) we obtain the following representations of $\varphi_{1}, \varphi_{2}$

$$
\begin{aligned}
\varphi_{1}(z)= & a_{0}^{\prime}+\sum_{k=1}^{\infty}\left(a_{k}^{\prime} \eta^{k} \cos k \theta-b_{k}^{\prime} \eta^{k} \sin k \theta\right) \quad \forall z=\eta \exp \{i \theta\} \in \mathrm{D}_{r}, \\
\varphi_{2}(z)= & a_{0}^{\prime \prime}+\sum_{k=1}^{\infty}\left(a_{k}^{\prime \prime} \eta^{k}+a_{-k}^{\prime \prime} \eta^{-k}\right) \cos k \theta \\
& +\sum_{k=1}^{\infty}\left(b_{-k}^{\prime \prime} \eta^{-k}-b_{k}^{\prime \prime} \eta^{k}\right) \sin k \theta \quad \forall z=\eta \exp \{i \theta\} \in \mathrm{A}_{r}
\end{aligned}
$$

Any pair of functions $\varphi=\left(\varphi_{1}, \varphi_{2}\right)$ given by $(3.7),(3.8)$, respectively, satisfy the first equation of the system (2.1) in the corresponding domains. The second condition of (2.1) and the formula (3.8) yields

$$
a_{0}^{\prime \prime}+\sum_{k=1}^{\infty}\left(a_{k}^{\prime \prime}+a_{-k}^{\prime \prime}\right) \cos k \theta+\sum_{k=1}^{\infty}\left(b_{-k}^{\prime \prime}-b_{k}^{\prime \prime}\right) \sin k \theta=-\cos \theta \quad \forall \theta \in[0,2 \pi] .
$$

Hence, by uniqueness of the Fourier series, coefficients of (3.8) satisfy the following relations

$$
\begin{aligned}
& a_{0}^{\prime \prime}=0, \quad a_{-1}^{\prime \prime}=-\left(1+a_{1}^{\prime \prime}\right), \\
& a_{-k}^{\prime \prime}=-a_{k}^{\prime \prime}, \quad k=2,3, \ldots, \quad b_{-k}^{\prime \prime}=b_{k}^{\prime \prime}, \quad k=1,2, \ldots
\end{aligned}
$$


Then we can rewrite the relation (3.8) in the following form

$$
\begin{aligned}
\varphi_{2}(z)= & \left(a_{1}^{\prime \prime} \eta-\left(1+a_{1}^{\prime \prime}\right) \eta^{-1}\right) \cos \theta+\sum_{k=2}^{\infty} a_{k}^{\prime \prime}\left(\eta^{k}-\eta^{-k}\right) \cos k \theta \\
& +\sum_{k=1}^{\infty} b_{k}^{\prime \prime}\left(\eta^{-k}-\eta^{k}\right) \sin k \theta \quad \forall z=\eta \exp \{i \theta\} \in \operatorname{cl} \mathrm{A}_{r},
\end{aligned}
$$

The third condition of (2.1) implies the equality

$$
\begin{aligned}
& a_{0}^{\prime}+\left(\left(a_{1}^{\prime}-a_{1}^{\prime \prime}\right) r+\left(1+a_{1}^{\prime \prime}\right) r^{-1}\right) \cos \theta+\sum_{k=2}^{\infty}\left(\left(a_{k}^{\prime}-a_{k}^{\prime \prime}\right) r^{k}+a_{k}^{\prime \prime} r^{-k}\right) \cos k \theta \\
& -\sum_{k=1}^{\infty}\left(\left(b_{k}^{\prime}-b_{k}^{\prime \prime}\right) r^{k}+b_{k}^{\prime \prime} r^{-k}\right) \sin k \theta=0 \quad \forall \theta \in[0,2 \pi] .
\end{aligned}
$$

Thus, we have

$$
\begin{aligned}
& a_{0}^{\prime}=0, \quad a_{1}^{\prime}=a_{1}^{\prime \prime}-\left(1+a_{1}^{\prime \prime}\right) r^{-2}, \\
& a_{k}^{\prime}=a_{k}^{\prime \prime}\left(1-r^{-2 k}\right), \quad k=2,3 \ldots, \quad b_{k}^{\prime}=b_{k}^{\prime \prime}\left(1-r^{-2 k}\right), \quad k=1,2, \ldots
\end{aligned}
$$

Hence, we can rewrite the representation (3.7) in the following form

$$
\begin{aligned}
\varphi_{1}(z)= & \eta\left(a_{1}^{\prime \prime}-\left(1+a_{1}^{\prime \prime}\right) r^{-2}\right) \cos \theta+\sum_{k=2}^{\infty} a_{k}^{\prime \prime} \eta^{k}\left(1-r^{-2 k}\right) \cos k \theta \\
& -\sum_{k=1}^{\infty} b_{k}^{\prime \prime} \eta^{k}\left(1-r^{-2 k}\right) \sin k \theta \quad \forall z=\eta \exp \{i \theta\} \in \operatorname{clD} r
\end{aligned}
$$

Taking into account, that normal derivatives on the circle centered at the origin coincide with the derivatives with respect to polar radius, we have the following relations $\frac{\partial \varphi_{k}}{\partial \mathbf{n}}=\left.\frac{\partial \varphi_{k}}{\partial \eta}\right|_{\eta=r}, k=1,2$. Hence, dividing by $\lambda_{1}+\lambda_{2}$ the last condition of (2.1) we obtain the following relation

$$
\begin{aligned}
& \left(-\rho a_{1}^{\prime \prime}+\left(1+a_{1}^{\prime \prime}\right) r^{-2}\right) \cos \theta-\rho \sum_{k=2}^{\infty} k a_{k}^{\prime \prime} r^{k-1}\left(1-r^{-2 k}\right) \cos k \theta \\
& -\sum_{k=1}^{\infty} k b_{k}^{\prime \prime} r^{k-1}\left(-\rho+r^{-2 k}\right)=0 \quad \forall \theta \in[0,2 \pi]
\end{aligned}
$$

where $\rho=\left(\lambda_{1}-\lambda_{2}\right) /\left(\lambda_{1}+\lambda_{2}\right)$. Let

$$
0<r<\min \left\{|\rho|^{-1 / 2}, 1\right\}
$$

then the following inequalities hold

$$
\left(1-r^{-2 k}\right) \neq 0, \quad k=2,3, \ldots, \quad-\rho+r^{-2 k} \neq 0, \quad k=1,2, \ldots,
$$

thus, the equality (3.12) is true for such $r$ if and only if the following relations hold

$$
a_{1}^{\prime \prime}=\rho^{-1}\left(r^{2}-\rho^{-1}\right)^{-1}, \quad a_{k}^{\prime \prime}=0, \quad k=2,3, \ldots, \quad b_{k}^{\prime \prime}=0, \quad k=1,2, \ldots
$$


Taking into account that for any $0<\lambda_{1}, \lambda_{2}<+\infty$ the Bergmann contrast parameter $\rho$ satisfies the inequality $-1<\rho<1$, clarify that the inequality (3.13) holds for any $r \in(0,1)$, therefore, relations (3.14) hold for all $r \in(0,1)$.

Then $1+a_{1}^{\prime \prime}=r^{2}\left(r^{2}-\rho^{-1}\right)^{-1}$ and (3.10) turns to $a_{1}^{\prime}=-\left(1-\rho^{-1}\right) \times$ $\left(r^{2}-\rho^{-1}\right)^{-1}$, therefore, the equality (3.11) coincides with the formula (3.1). By using the last relations, we obtain that (3.9) coincides with (3.2). Formulas (3.3) and (3.4) are simple consequences of the continuity of the functions (3.1) and (3.2), accordingly, in $\mathrm{clD}_{r}$ and $\mathrm{cl} \mathrm{A}_{r}$. Therefore, the pair of functions (3.1) and (3.2) defines the unique solution of the problem (2.1).

\section{Effective Conductivity}

In the considered case $x$-component $F^{x}$ of the complete flux can be normalized as follows $F^{x}=-\pi$ (see [8]).

Theorem 2. Under assumptions of Theorem 1 the following formula holds

$$
\lambda_{e f f}^{x}(r)=\lambda_{2} \frac{\rho^{-1}+r^{2}}{\rho^{-1}-r^{2}} .
$$

Furthermore, the function (2.3) is the sum of the following series

$$
\lambda_{e f f}^{x}(r)=\lambda_{2}\left(1+\rho r^{2}\right)\left(1+\sum_{k=1}^{\infty} \rho^{k} r^{2 k}\right)
$$

converging for any $0<r<1$.

Proof. By the Green formula

$$
\begin{aligned}
\lambda_{e f f}^{x}(r) & =-\pi^{-1}\left(\lambda_{1} \oint_{\gamma_{r}} \varphi_{1}(x, y) d y+\lambda_{2}\left(\oint_{\Gamma}-\oint_{\gamma_{r}}\right) \varphi_{2}(x, y) d y\right) \\
& =-\pi^{-1}\left(\oint_{\gamma_{r}}\left(\lambda_{1} \varphi_{1}(x, y)-\lambda_{2} \varphi_{2}(x, y)\right) d y+\lambda_{2} \oint_{\Gamma} \varphi_{2}(x, y) d y\right),
\end{aligned}
$$

where in the contour integrals the orientation is assumed to be the opposite to the clockwise direction, $\varphi_{1}$ ( or $\varphi_{2}$ ) is a restriction of $\varphi$ to $\mathrm{D}_{r}$ ( or $\mathrm{A}_{r}$ ). Therefore, by the formulas (3.3) and (3.4) and using the polar coordinates $(\eta, \theta)$ we rewrite the last equality in the following form

$$
\lambda_{e f f}^{x}(r)=-\pi^{-1} \frac{\lambda_{2} \rho^{-1}-\left(\lambda_{2} \rho^{-1}+\lambda_{1}\left(1-\rho^{-1}\right)\right) r^{2}}{-\rho^{-1}+r^{2}} \int_{0}^{2 \pi}(\cos \theta)^{2} d \theta .
$$

Since

$$
\lambda_{2} \rho^{-1}+\lambda_{1}\left(1-\rho^{-1}\right)=-\lambda_{2}, \quad \int_{0}^{2 \pi}(\cos \theta)^{2} d \theta=\pi,
$$

the equality (4.3) turns to the formula (4.1), which holds for all $r \in(0,1)$ ).

Then the equality (4.2) follows from the obtained formula after substitution to it the following series expansion

$$
\frac{1}{-\rho^{-1}+r^{2}}=-\rho-\rho \sum_{k=1}^{\infty} \rho^{k} r^{2 k} \quad \forall r \in(0,1)
$$


Corollary 1. $\lambda_{\text {eff }}^{x}(r)$ is an even function in $r$, continuous for all $r \in[0,1]$. Furthermore, $\lambda_{\text {eff }}^{x}(1)=\lambda_{1}, \lambda_{\text {eff }}^{x}(0)=\lambda_{2}$.

Remark 1. The obtained formula (4.1) coincides with the celebrated ClausisMossotti approximation formula for $x$-component of the effective conductivity tensor (see, e.g. [8]).

Remark 2. If one have to construct the composite material with a prescribed value of $\lambda_{\text {eff }}^{x}(r)$, then formula (4.1) gives an exact relation between values of conductivity of matrix and inclusion (in the case of fixed $r$ ) or a functional representation of $\lambda_{2}$ in terms of $r$ (in the case of fixed $\lambda_{1}$ ).

\section{Acknowledgements}

The authors are thankful to Prof. M. Lanza de Cristoforis for hospitality during their stay at the Department of Mathematics, Padova University. The first author is partially supported by the Grant of Ministry of Education, Science, Youth and Sports of Ukraine, Project No. 0112U000374. The authors are grateful to Prof. Raimondas Čiegis for his support of the presented research and to anonymous referees for the valuable discussion of the results of the paper.

\section{References}

[1] D.J. Bergmann. The dielectric constants of a composite material - a problem in classical physics. Phys. Rep. C, 43:377-407, 1978. http://dx.doi.org/10.1016/0370-1573(78)90009-1.

[2] A. Cherkaev. Variational Methods for Structural Optimization. Springer-Verlag, New York, 2000.

[3] A. Cherkaev and A. Pruss. Effective conductivity of spiral and other radial symmetric assemblages. arXiv:1206.3604v2 [math-ph], 2012.

[4] I.I. Komyak and V.V. Mityushev. On the solution of a general boundary value problem of linear conjugation for an annulus. Izv. Akad. Nauk BSSR, Ser. Fiz.Mat. Nauk, 3:25-33, 1983. (In Russian)

[5] G.W. Milton. The Theory of Composites. Cambridge University Press, 2004.

[6] V.V. Mityushev. Solution of $\mathbb{R}$-linear conjugation problem (markushevich problem) for a ring in a special case. Sov. Mat., 30(3):92-95, 1986. Translation from Izv. Vyssh. Uchebn. Zaved., Mat. 3:67-69, 1986. (In Russian)

[7] V.V. Mityushev. Conductivity of a two-dimensional composite containing elliptical inclusions. Proc. R. Soc. A, 465:2991-3010, 2009.

[8] V.V. Mityushev and S.V. Rogosin. Constructive Methods to Linear and NonLinear Boundary Value Problems of the Analytic Function. Theory and Applications. Chapman \& Hall/CRC Monogr. Surv. Pure Appl. Math. Chapman \& Hall/CRC, 2000.

[9] N.I. Muskhelishvili. On the problem of torsion and bending of elastic beams composed of different materials. Izvestiya Akad. Nauk SSSR., Ser. Mat. and Nat. Sci., 7:907-945, 1932. (In Russian) 
[10] I.N. Vekua and A.K. Rukhadze. The problem of the torsion of a circular cylinder reinforced by transversal circular beam. Izvestiya Akad. Nauk SSSR., Ser. Mat. and Nat. Sci., 3:373-386, 1933. (In Russian)

[11] I.N. Vekua and A.K. Rukhadze. Torsion and transversal bending of the beam compounded by two materials restricted by confocal ellipses. Prikladnaya Matematika i Mechanika (Leningrad), 1:167-178, 1933. (In Russian) 